\title{
The Impact of Atopic Dermatitis on Children's Sleep Duration and Their Mother's Suicidal Ideation
}

\author{
Kim Ji Young ${ }^{1}$ \& Yoon Heun Keung ${ }^{2}$ \\ ${ }^{1}$ Department of Nursing, Wonkwang Health Science University, Iksan, South Korea \\ 2 Department of Nursing, Howon University, Gunsan, South Korea \\ Correspondence: Yoon Heun Keung, Department of Nursing, Howon University, 64 Howondae 3gil, Impi, \\ Gunsan-Si, Jeollabuk-do, 54058, South Korea. Tel: 82-63-450-7766. E-mail: yhk@hanmail.net
}

Received: December 3, 2017 Accepted: December 26, 2017 Online Published: January 8, 2018

doi:10.5539/gjhs.v10n2p115 URL: https://doi.org/10.5539/gjhs.v10n2p115

\begin{abstract}
Purpose: This study was performed to identify the effects of child's atopic dermatitis (AD) on sleep duration and their moder's suicidal ideation.

Methods: The subjects were 2,845 mothers of preadolescent and adolescent children who participated in the $6^{\text {th }}$ Korea National Health and Nutrition Examination Survey (KNHANES VI; 2013 and 2015). The data gathered were analyzed with a chi-square test and logistic regression using the SPSS 18.0 program for Windows.

Results: With respect to the effect of a child's AD on maternal sleep duration and suicidal ideation, sleep duration was statistically and significantly lower in mothers of children with $A D(O R=1.477$ in Model $1, O R=1.552$ in Model 2 and $\mathrm{OR}=1.547$ in Model 3) than in mothers of children without AD. ) Although not statistically significant, suicidal ideation was higher in mothers of affected children $(\mathrm{OR}=1.348$ in Model 1, OR=1.414, $p=.252$ in Model 2 and $\mathrm{OR}=1.109$ in Model 3) than in mothers of non-affected children.

Conclusion: Mothers of children affected by atopic dermatitis suggest the development of nursing interventions for health maintenance because they can not rule out the possibility of depression or suicidal thoughts due to sleep disorders.
\end{abstract}

Keywords: atopic dermatitis, sleep duration, suicidal ideation, mothers, children

\section{Introduction}

Atopic dermatitis (AD) is a chronic inflammatory skin disorder characterized by a relapsing course, severe pruritus and eczematous lesions. Its prevalence is higher among children and adolescents (15.1\%) than adults (3.4\%) (Ministry of Health and Welfare, 2015; Draaisma, 2003). AD is a common condition in childhood and adolescence, and typically disappears by about 3 years of age. However, AD persists until adulthood in 17-70\% of affected patients, and thereby posing a burden on patients and their families (Lee, 2003). Furthermore, this condition poses substantial economic burden and loss to the country and, in 2015, the annual medical care expenditure per person had increased by $23.2 \%$ since 2010 (National Health Insurance Service, 2016).

The pathogenesis or cause of the disease has not yet been determine. However, AD arises because of a complex interaction of internal and external factors including genetic risk and the activation of congenital and acquired immune systems (Devereux \& Seaton, 2005). AD is the first clinical manifestation of atopic march which progresses to asthma or allergic rhinitis. Among genetic factors, a family history of allergic disease is the leading risk factor (Pyun, 2005), and parents have been found to experience psychological distress because of continuous monitoring of skin lesions for symptom management (Basra \& Finlay, 2007).

Skin infection recurs by scratching the affected area of skin due to pruritus, one of the key diagnostic criteria of AD. Furthermore, sleep disturbance is associated with intense pruritus worsening at night (Williams, 1999). Chronic disease progression in children requires considerable parental care and, due to this, parents can experience very high levels of anxiety and depression (van Oers, 2014). Parents sleep with their children at night to prevent pruritus-induced scratching which may lead to bleeding and infection. Sleep deprivation associated with frequent waking (due to pruritus) causes fatigue in parents and affects their ability to perform activities of daily living (Lewandowski, Ward, \& Palermo, 2011). Parental quality of life can be influenced by even mild AD symptoms 
over time, and is substantially affected by aggravation of symptoms (Lifschitz, 2015). According to a study on familial quality of life of patients with skin disease, families were found to be particularly influenced emotionally and $98 \%$ of them suffered emotionally from disease progression (Basra \& Finlay, 2007).

Suicide is considered an extreme behavior initiated when overall life satisfaction decreases or there are difficulties in finding solutions to problems faced (Korea Statistical Information Service, 2015). Prevalence of suicidal ideation in patients with $\mathrm{AD}$, between the ages of 15 and 49 years, was $0.21 \%, 6.0 \%$, and $19.6 \%$ in mild, moderate and severe conditions, respectively. Suicidal impulse rates were $0.11 \%, 0.35 \%$, and $3.28 \%$ according to mild, moderate and severe degrees of severity among parents with $\mathrm{AD}$ children at ages younger than 14 years (Kimata, 2006).

In 2014, a newspaper, the Kyunghyang Shinmun, reported on a mother of an 8-year old child with AD who killed her child and then committed suicide after suffering from aggravated symptoms in Korea (Kwon, 2014). In 2004, the Mainichi Shimbun reported that a mother killed her 4-year old child and herself due to despair from relapsing disease and sleep deprivation caused by the baby's intensified fretfulness at night (Kimata, 2006). These tragic incidents were reported because double suicides took place as parents chose to commit suicide due to a feeling of guilt after child's death.

Sleep duration and suicidal ideation of mothers, the primary caregivers, of children with AD have rarely been reported in literature. Suicidal ideation of parents has been addressed in a number of international studies on the social impact of children's AD and sleep disturbances in themselves and their parents (Lapidus \& Kerr, 2001) as well as in patients with AD (Kimata, 2006). Primarily, the majority of previous studies have explored prognostic factors of the quality of life of parents with children affected by AD (Warschburger, Buchholz, \& Petermann, 2004) and the effect of AD on children and their families (Chamlin, Frieden, Williams, \& Chren, 2004). A Korean study on the effect of child's AD on the mental health of primary caregivers has investigated the association between child's AD and suicidal ideation of mothers (Choi \& Lee, 2017). Other studies have examined stress in parents of children with AD (Yoo \& Kim, 2004; Kim, 2011), and the difficulties and needs of parents of children with AD (Yoon \& Bae, 2013).

The presence of a chronic disease easily disturbs the sleep of mothers, and they may have difficulties in managing their own emotions with the progression of their child's condition. For these reasons, a mother's psychological state requires special attention. This study was performed to provide a reference base to devise measures for reducing potential risk factors of $\mathrm{AD}$ and improve mothers' physical and mental health by improving social support and developing early intervention plans through an overall assessment of the impact of disease and treatment on family members.

This study intends to investigate the effects of child's atopic dermatitis (AD) on sleep time and suicidal ideation of mothers. The detailed purposes are as follows: - To identify differences in the general characteristics and health-related traits of mothers between $\mathrm{AD}$ and non-AD groups

- To identify differences in sleep duration according to subjects' characteristics

- To identify differences in suicidal ideation according to subjects' characteristics

- To identify the impact of child's AD on sleep duration and suicidal ideation of mothers

\section{Method}

This study is a secondary analysis of the data from the $6^{\text {th }}$ Korea National Health and Nutrition Examination Survey (KNHANES VI; 2013 and 2015) to explore the effects of child's AD on maternal sleep duration and suicidal ideation among children (between the ages of 1 and 18 years). with AD This study excluded suicide-related items from the 2014 KNHANES because the survey included data on adolescents aged 12 to 18 years.

\subsection{Participant (Subject) Characteristics}

General characteristics of subjects were age, marital status, educational level, household income and occupational status. Age was calculated using Western age. Marital status was categorized into married (co-resident/separated), divorced, or widowed. Education level was classified into elementary school graduates or below, junior high school graduates, high school graduates and college graduates or above. Household income level was determined by dividing sample households and populations into four groups. Occupational status was divided into two categories, employed and unemployed.

Health-related traits of mothers were smoking and drinking status, subjective health status, hypertension, hyperlipidemia, asthma, depression, $\mathrm{AD}$, allergic rhinitis and stress perception rate. Drinking status was grouped 
into non-drinkers and current drinkers. Smoking status was divided into current smokers and non-smokers. Subjective health status was categorized into healthy, moderately healthy, and unhealthy. The presence of each disease was determined based on a medical diagnosis. Stress perception rate was grouped into either little to no stress or a lot of stress while performing activities of daily living.

Sleep duration was evaluated using answers to the survey question,'How many hours do you usually sleep a day?'. Suicidal ideation was categorized into either 'yes' or 'no' to the question, 'I have earnestly thought about committing suicide within the past year'. Since this 2013 survey question has been changed to 'I have seriously thought about committing suicide within the past year' in 2015, it is recommended not to compare results by year considering differences in answers. However, this study used both questions in analyzing suicidal ideation.

\subsection{Sampling Procedures}

The subject pool was comprised of 2,845 mothers (442 in the AD group and 2,403 in the non-AD group) of 3,085 children (486 with AD and 2,599 without AD) between the ages of 1 and 18 years among the15,398 individuals who participated in the KNHANES (2013 and 2015).

\subsubsection{Research Design}

The data sets were analyzed using the SPSS 18.0 program for Windows. A complex chi-square test was performed to identify differences in general characteristics and health-related traits between the $\mathrm{AD}$ and non-AD groups. Complex samples logistic regression was done to determine the effects of child's AD on sleep duration and suicidal ideation of mothers.

\section{Results}

\subsection{General Characteristics and Health-Related Traits of AD and Non-AD Groups}

A total of 2,845 mothers were divided into two groups: 442 in the AD group and 2,403 in the non-AD group. In this study, maternal age in the $\mathrm{AD}$ group ranged between 40-49 (16.4\%) and 30-39 (16.1\%), implying that this seems to be associated with child's ages ranging within pediatric and adolescent periods. In regards to marital status, the percentages of divorced (18.3\%) and widowed (25.6\%) mothers were higher in the AD group, indicating that $43.9 \%$ of mothers were raising children alone. According to mothers' general characteristics and health-related traits, variables showing differences between $\mathrm{AD}$ and non- $\mathrm{AD}$ groups were asthma $\left(\chi^{2}=13.464, \mathrm{p}=.003\right), \mathrm{AD}\left(\chi^{2}=26.052\right.$, $\mathrm{p}<.001)$ and allergic rhinitis $\left(\chi^{2}=7.546, \mathrm{p}=.018\right)$. Thus, mothers in the AD group were more likely to have asthma $(33.9 \%), \mathrm{AD}(44.8 \%)$ and allergic rhinitis $(20.2 \%)$ (Table 1$)$.

Table 1. Differences of general characteristics and health related characteristics between mothers of atopic group and mothers of non-atopic group $(N=2,845)$

\begin{tabular}{|c|c|c|c|c|c|}
\hline \multirow[t]{2}{*}{ Characteristics } & \multirow[t]{2}{*}{ Categories } & $\begin{array}{l}\text { Atopic group } \\
(n=442)\end{array}$ & $\begin{array}{l}\text { Non-atopic group } \\
(n=2,403)\end{array}$ & \multirow[t]{2}{*}{$\chi^{2}$} & \multirow[t]{2}{*}{$p$} \\
\hline & & Weighted n $(\%)$ & Weighted n (\%) & & \\
\hline \multirow{4}{*}{ Age(year) } & $20 \sim 29$ & $9(10.6)$ & $87(89.4)$ & \multirow{4}{*}{6.177} & \multirow{4}{*}{.203} \\
\hline & $30 \sim 39$ & $213(16.1)$ & $1,128(83.9)$ & & \\
\hline & $40 \sim 49$ & 209 (16.4) & $1,092(83.6)$ & & \\
\hline & $\geq 50$ & $11(9.5)$ & $96(90.5)$ & & \\
\hline \multirow{3}{*}{ Marital status } & Spouse & $419(15.6)$ & 2,297 (84.4) & \multirow{3}{*}{2.563} & \multirow{3}{*}{.472} \\
\hline & Divorced & $17(18.3)$ & $83(81.7)$ & & \\
\hline & Widowed & $6(25.6)$ & $16(74.4)$ & & \\
\hline \multirow{4}{*}{ Education } & $\leq$ Elementary school & $2(4.3)$ & $42(95.7)$ & \multirow{4}{*}{6.008} & \multirow{4}{*}{.246} \\
\hline & Middle school & $10(15.1)$ & $64(84.9)$ & & \\
\hline & High school & $192(17.1)$ & 973 (82.9) & & \\
\hline & $\geq$ College & $215(15.8)$ & $1,152(84.2)$ & & \\
\hline
\end{tabular}




\begin{tabular}{|c|c|c|c|c|c|}
\hline \multirow{4}{*}{ Household income } & Q1(Lowest) & $29(14.9)$ & $195(85.1)$ & \multirow{4}{*}{0.491} & \multirow{4}{*}{.953} \\
\hline & Q2 & $120(15.1)$ & $666(84.9)$ & & \\
\hline & Q3 & $149(16.0)$ & $802(84.0)$ & & \\
\hline & Q4(Highest) & $141(16.1)$ & $729(83.9)$ & & \\
\hline \multirow{2}{*}{ Occupation } & Yes & $222(15.6)$ & $1,208(84.4)$ & \multirow{2}{*}{0.660} & \multirow{2}{*}{.467} \\
\hline & No & $196(16.8)$ & $1,025(83.2)$ & & \\
\hline \multirow{2}{*}{ Drinking } & Present drinker & $389(15.7)$ & $2,116(84.3)$ & \multirow{2}{*}{0.362} & \multirow{2}{*}{.608} \\
\hline & Never drunk & $32(17.3)$ & $166(82.7)$ & & \\
\hline \multirow{2}{*}{ Smoking } & Present smoker & $19(16.7)$ & $119(83.3)$ & \multirow{2}{*}{0.001} & \multirow{2}{*}{.983} \\
\hline & Never smoked & $32(16.6)$ & $144(83.4)$ & & \\
\hline \multirow{3}{*}{$\begin{array}{l}\text { Self-reported } \\
\text { health status }\end{array}$} & Healthy & $143(14.7)$ & $835(85.3)$ & \multirow{3}{*}{7.786} & \multirow{3}{*}{.069} \\
\hline & Average & $213(15.8)$ & $1,145(84.2)$ & & \\
\hline & Unhealthy & $63(21.1)$ & $257(78.9)$ & & \\
\hline \multirow{2}{*}{ Hypertension } & Yes & $6(8.8)$ & $70(91.2)$ & \multirow{2}{*}{3.585} & \multirow{2}{*}{.139} \\
\hline & No & $415(16.3)$ & $2,178(83.7)$ & & \\
\hline \multirow{2}{*}{ Hyperlipidemia } & Yes & $18(13.6)$ & $101(86.4)$ & \multirow{2}{*}{0.658} & \multirow{2}{*}{.542} \\
\hline & No & $403(16.2)$ & $2,147(83.8)$ & & \\
\hline \multirow{2}{*}{ Asthma } & Yes & $19(33.9)$ & $40(66.1)$ & \multirow{2}{*}{13.464} & \multirow{2}{*}{.003} \\
\hline & No & $400(15.7)$ & $2,196(84.3)$ & & \\
\hline \multirow{2}{*}{ Depression } & Yes & $14(16.5)$ & $85(83.5)$ & \multirow{2}{*}{0.014} & \multirow{2}{*}{.920} \\
\hline & No & $405(16.1)$ & $2,151(83.9)$ & & \\
\hline \multirow{2}{*}{ Atopic dermatitis } & Yes & $18(44.8)$ & $25(55.2)$ & \multirow{2}{*}{26.052} & \multirow{2}{*}{$<.001$} \\
\hline & No & $401(15.7)$ & $2,211(84.3)$ & & \\
\hline \multirow{2}{*}{ Allergic rhinitis } & Yes & $98(20.2)$ & $416(79.8)$ & \multirow{2}{*}{7.546} & \multirow{2}{*}{.018} \\
\hline & No & $321(15.2)$ & $1,820(84.8)$ & & \\
\hline & Rarely & $300(15.5)$ & $1,671(84.5)$ & & \\
\hline Stress & Often & $121(16.7)$ & $611(83.3)$ & 0.541 & .348 \\
\hline
\end{tabular}

\subsection{Differences in Sleep Duration According to Subject Characteristics}

Of the 2,845 mothers in this study, the average sleep duration was less than five hours a day in the AD group (16.5\%) compared to the non-AD group (11.8\%). The number of mothers sleeping the recommended 6-8 hours was found to be lower in the $\mathrm{AD}$ group (75.8\%) than in the non-AD group (82.6\%). Variables showing differences in sleep duration were age $\left(\chi^{2}=63.531, \mathrm{p}=<.001\right)$, marital status $\left(\chi^{2}=62.497, \mathrm{p}=<.001\right)$, educational level $\left(\chi^{2}=37.977, \mathrm{p}=.010\right)$, household income $\left(\chi^{2}=39.955, \mathrm{p}=.013\right)$, occupation $\left(\chi^{2}=23.542, \mathrm{p}=.006\right)$, subjective health status $\left(\chi^{2}=37.994, p=.005\right)$, hypertension $\left(\chi^{2}=32.720, p=.006\right)$ and stress perception rate $\left(\chi^{2}=41.104, p=<.001\right)$.

Sleep duration of less than five hours was highest in mothers aged 50 or above $(24.0 \%)$, followed by those aged $30-49$ years $(24.2 \%)$. This study has determined that recent trends in the delay of first marriage have led to an increase in the average age of mothers. Mothers with divorced marital status, an educational level of less than elementary school, low income level, occupation, poor subjective health status, hypertension and a lot of stress in daily living were identified to sleep an average of less than 5 hours a day (Table 2). 
Table 2. Differences of characteristics sleep duration of study population $(N=2,845)$

\begin{tabular}{|c|c|c|c|c|c|c|}
\hline \multirow{4}{*}{ Characteristics } & \multirow{4}{*}{ Categories } & \multicolumn{3}{|c|}{ Sleep duration(hr/day) } & \multirow[t]{4}{*}{$\chi^{2}$} & \multirow{4}{*}{$p$} \\
\hline & & $\leq 5$ & $6 \sim 8$ & $\geq 9$ & & \\
\hline & & weighted & weighted & Weighted & & \\
\hline & & $n(\%)$ & $n(\%)$ & $n(\%)$ & & \\
\hline \multirow{2}{*}{ Atopic group } & $\mathrm{Yes}(\mathrm{N}=442)$ & $58(16.5)$ & $335(75.8)$ & $28(7.7)$ & \multirow{2}{*}{10.991} & \multirow{2}{*}{.033} \\
\hline & $\mathrm{No}(\mathrm{N}=2,403)$ & $248(11.8)$ & $1,903(82.6)$ & $131(5.6)$ & & \\
\hline \multirow{4}{*}{ Age(year) } & $20 \sim 29$ & $5(3.1)$ & $70(79.0)$ & $15(18.0)$ & \multirow{4}{*}{63.531} & \multirow{4}{*}{$<.001$} \\
\hline & $30 \sim 39$ & $128(10.4)$ & $1,061(82.0)$ & $91(7.6)$ & & \\
\hline & $40 \sim 49$ & $153(13.8)$ & $1,038(82.3)$ & $48(3.9)$ & & \\
\hline & $\geq 50$ & $21(24.0)$ & $83(72.2)$ & $5(3.8)$ & & \\
\hline \multirow{3}{*}{ Marital status } & Spouse & $273(11.5)$ & $2,174(82.8)$ & $146(5.6)$ & \multirow{3}{*}{62.497} & \multirow{3}{*}{$<.001$} \\
\hline & Divorced & $30(33.2)$ & $57(54.5)$ & $10(12.3)$ & & \\
\hline & Widowed & $3(12.9)$ & $17(84.0)$ & $1(3.1)$ & & \\
\hline \multirow{4}{*}{ Education } & $\leq$ Elementary school & $11(25.7)$ & $32(67.1)$ & $3(7.2)$ & \multirow{4}{*}{37.977} & \multirow{4}{*}{.010} \\
\hline & Middle school & $10(20.4)$ & $54(67.4)$ & $10(12.2)$ & & \\
\hline & High school & $160(14.9)$ & 935 (78.9) & $70(6.2)$ & & \\
\hline & $\geq$ College & $121(9.4)$ & $1,175(85.1)$ & $75(5.5)$ & & \\
\hline \multirow{4}{*}{$\begin{array}{l}\text { Household } \\
\text { income }\end{array}$} & Q1(Lowest) & $41(22.8)$ & $150(66.5)$ & $20(10.8)$ & \multirow{4}{*}{39.955} & \multirow{4}{*}{.013} \\
\hline & Q2 & $84(12.1)$ & $610(81.9)$ & $49(6.0)$ & & \\
\hline & Q3 & $98(11.9)$ & $784(83.2)$ & $44(4.9)$ & & \\
\hline & Q4(Highest) & $80(10.4)$ & $703(84.2)$ & $43(5.4)$ & & \\
\hline \multirow{2}{*}{ Occupation } & Yes & $179(13.7)$ & $1,192(82.2)$ & $63(4.1)$ & \multirow{2}{*}{23.542} & \multirow{2}{*}{.006} \\
\hline & No & $123(11.2)$ & $1,004(80.4)$ & $95(8.4)$ & & \\
\hline \multirow{2}{*}{ Drinking } & Present drinker & $276(12.1)$ & $2,095(81.9)$ & $149(6.0)$ & \multirow{2}{*}{3.921} & \multirow{2}{*}{.431} \\
\hline & Never drunk & $31(16.9)$ & $157(77.6)$ & $10(5.5)$ & & \\
\hline \multirow{2}{*}{ Smoking } & Present smoker & $29(21.6)$ & $90(63.3)$ & $19(15.1)$ & 4168 & 425 \\
\hline & Never smoked & $26(17.2)$ & $132(73.5)$ & $19(9.3)$ & 4.100 & .425 \\
\hline & Healthy & $71(8.2)$ & $856(86.9)$ & $52(4.9)$ & & \\
\hline $\begin{array}{l}\text { Self-reported } \\
\text { health status }\end{array}$ & Average & $179(14.1)$ & $1,101(79.6)$ & $81(6.3)$ & 37.994 & .005 \\
\hline & Unhealthy & $52(18.3)$ & $244(73.4)$ & $25(8.2)$ & & \\
\hline Hun & Yes & $17(25.4)$ & $50(58.7)$ & $8(15.9)$ & 22720 & 006 \\
\hline пурептетвіолі & No & $285(12.0)$ & $2,161(82.3)$ & $150(5.7)$ & 32.120 & .000 \\
\hline Humelinidomia & Yes & $20(20.6)$ & $91(74.4)$ & $5(5.0)$ & 0006 & 150 \\
\hline пуреппрісенпла & No & $282(12.0)$ & $2,120(81.9)$ & $153(6.1)$ & 9.000 & .150 \\
\hline$A$ & Yes & $6(11.0)$ & $48(81.0)$ & $5(8.0)$ & 0.157 - & 007 \\
\hline Asthma & No & $296(2.6)$ & $2,152(81.4)$ & $153(6.0)$ & $0.45 /$ & .881 \\
\hline Donroccion & Yes & 19 (21.9) & $69(67.0)$ & $10(11.2)$ & 15260 & 052 \\
\hline 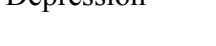 & No & $283(12.2)$ & $2,131(82.0)$ & $148(5.8)$ & & \\
\hline
\end{tabular}




\begin{tabular}{lllllll}
\hline \multirow{2}{*}{ Atopic dermatitis } & Yes & $4(6.8)$ & $35(85.1)$ & $4(8.1)$ & 1.492 & .613 \\
& No & $298(12.6)$ & $2,165(81.3)$ & $154(6.0)$ & & \\
\hline \multirow{2}{*}{ Allergic rhinitis } & Yes & $69(14.7)$ & $421(79.5)$ & $25(5.8)$ & 2.603 & .567 \\
& No & $233(12.0)$ & $1,779(81.8)$ & $133(6.1)$ & & \\
\hline \multirow{2}{*}{ Stress } & Rarely & $177(10.0)$ & $1,698(84.4)$ & $109(5.6)$ & 41.104 & $<.001$ \\
& Often & $130(18.7)$ & $554(74.5)$ & $50(6.8)$ & & \\
\hline
\end{tabular}

\subsection{Difference in Suicidal Ideation According to Subject Characteristics}

Of all 2,845 mothers, the difference found in the suicidal ideation rate between $\mathrm{AD}(20.1 \%)$ and non-AD (15.7\%) groups $\left(\chi^{2}=1.287, p=.317\right)$ was not statistically significant even though the suicidal ideation rate was higher in the $\mathrm{AD}$ group. Variables indicating differences in suicidal ideation were marital status $\left(\chi^{2}=45.315, \mathrm{p}=<.001\right)$, household income $\left(\chi^{2}=30.638, p=.004\right)$, subjective health status $\left(\chi^{2}=29.476, p=.002\right)$, hyperlipidemia $\left(\chi^{2}=18.149\right.$, $\mathrm{p}=.010)$, depression $\left(\chi^{2}=141.706, \mathrm{p}=<.001\right)$, allergic rhinitis $\left(\chi^{2}=29.220, \mathrm{p}=.001\right)$, and stress perception rate $\left(\chi^{2}=107.394, \mathrm{p}=<.001\right)$.

Mothers with divorced marital status, low household income level, poor subjective health status, hyperlipidemia, depression, allergic rhinitis and a lot of stress in daily living had a significantly higher suicidal ideation rate (Table $3)$.

Table 3. Difference of characteristics suicidal ideation of study population $(N=2,845)$

\begin{tabular}{|c|c|c|c|c|c|}
\hline \multirow{4}{*}{ Characteristics } & \multirow{4}{*}{ Categories } & \multicolumn{2}{|c|}{ Suicidal ideation } & \multirow{4}{*}{$\chi^{2}$} & \multirow{4}{*}{$p$} \\
\hline & & Yes & No & & \\
\hline & & weighted & weighted & & \\
\hline & & $n(\%)$ & $n(\%)$ & & \\
\hline \multirow{2}{*}{ Atopic group } & $\operatorname{Yes}(\mathrm{N}=442)$ & $16(20.1)$ & $76(79.9)$ & \multirow{2}{*}{1.287} & \multirow{2}{*}{.317} \\
\hline & $\mathrm{No}(\mathrm{N}=2,403)$ & $405(15.7)$ & $2,206(84.3)$ & & \\
\hline \multirow{4}{*}{ Age(year) } & $20 \sim 29$ & $5(4.5)$ & $85(95.5)$ & \multirow{4}{*}{14.894} & \multirow{4}{*}{.057} \\
\hline & $30 \sim 39$ & $48(4.0)$ & $1,232(96.0)$ & & \\
\hline & $40 \sim 49$ & $30(2.4)$ & $1,209(97.6)$ & & \\
\hline & $\geq 50$ & $9(8.1)$ & $100(91.9)$ & & \\
\hline \multirow{3}{*}{ Marital status } & Spouse & $75(2.8)$ & $2,518(97.2)$ & \multirow{3}{*}{45.315} & \multirow{3}{*}{$<.001$} \\
\hline & Divorced & $14(14.2)$ & $83(85.8)$ & & \\
\hline & Widowed & $1(6.1)$ & $20(93.9)$ & & \\
\hline \multirow{4}{*}{ Education } & $\leq$ Elementary school & $6(12.8)$ & $40(87.2)$ & \multirow{4}{*}{14.745} & \multirow{4}{*}{.056} \\
\hline & Middle school & $3(2.9)$ & $71(97.1)$ & & \\
\hline & High school & $45(3.7)$ & $1,120(96.3)$ & & \\
\hline & $\geq$ College & $36(2.9)$ & $1,335(97.1)$ & & \\
\hline \multirow{4}{*}{ Household income } & Q1(Lowest) & $18(7.8)$ & $193(92.2)$ & \multirow{4}{*}{30.638} & \multirow{4}{*}{.004} \\
\hline & Q2 & $39(5.3)$ & 704 (94.7) & & \\
\hline & Q3 & $19(2.1)$ & 907 (97.9) & & \\
\hline & Q4(Highest) & $16(2.0)$ & $810(98.0)$ & & \\
\hline Occupation & Yes & $46(3.5)$ & $1,388(96.5)$ & 0.077 & .858 \\
\hline
\end{tabular}




\begin{tabular}{|c|c|c|c|c|c|}
\hline & No & $44(3.3)$ & $1,178(96.7)$ & & \\
\hline \multirow{2}{*}{ Drinking } & Present drinker & $84(3.3)$ & $2,436(96.7)$ & \multirow{2}{*}{0.581} & \multirow{2}{*}{.595} \\
\hline & Never drunk & $8(4.4)$ & $190(95.6)$ & & \\
\hline \multirow{2}{*}{ Smoking } & Present smoker & $13(9.6)$ & $125(90.4)$ & \multirow{2}{*}{3.164} & \multirow{2}{*}{.272} \\
\hline & Never smoked & $11(4.5)$ & $166(95.5)$ & & \\
\hline \multirow{3}{*}{$\begin{array}{l}\text { Self-reported health } \\
\text { status }\end{array}$} & Healthy & $19(1.8)$ & $960(98.2)$ & \multirow{3}{*}{29.476} & \multirow{3}{*}{.002} \\
\hline & Average & $46(3.4)$ & $1,315(96.6)$ & & \\
\hline & Unhealthy & $25(8.0)$ & $296(92.0)$ & & \\
\hline \multirow{2}{*}{ Hypertension } & Yes & $4(5.8)$ & $71(94.2)$ & \multirow{2}{*}{1.581} & \multirow{2}{*}{.380} \\
\hline & No & $86(3.3)$ & $2,510(96.7)$ & & \\
\hline \multirow{2}{*}{ Hyperlipidemia } & Yes & $11(9.8)$ & $105(90.2)$ & \multirow{2}{*}{18.149} & \multirow{2}{*}{.010} \\
\hline & No & $79(3.1)$ & $2,476(96.9)$ & & \\
\hline \multirow{2}{*}{ Asthma } & Yes & $4(8.1)$ & $55(91.9)$ & \multirow{2}{*}{3.792} & \multirow{2}{*}{.263} \\
\hline & No & $86(3.3)$ & $2,515(96.7)$ & & \\
\hline \multirow{2}{*}{ Depression } & Yes & $25(24.1)$ & $73(75.9)$ & \multirow{2}{*}{141.706} & \multirow{2}{*}{$<.001$} \\
\hline & No & $65(2.6)$ & $2,497(97.4)$ & & \\
\hline \multirow{2}{*}{ Atopic dermatitis } & Yes & $4(10.4)$ & $39(89.6)$ & \multirow{2}{*}{6.293} & \multirow{2}{*}{.106} \\
\hline & No & $86(3.3)$ & $2,531(96.7)$ & & \\
\hline \multirow{2}{*}{ Allergic rhinitis } & Yes & $34(7.4)$ & $481(92.6)$ & \multirow{2}{*}{29.220} & \multirow{2}{*}{.001} \\
\hline & No & $56(2.5)$ & $2,089(97.5)$ & & \\
\hline \multirow{2}{*}{ Stress } & Rarely & $24(1.1)$ & $1,960(98.9)$ & \multirow{2}{*}{107.394} & \multirow{2}{*}{$<.001$} \\
\hline & Often & $68(9.1)$ & $666(90.0)$ & & \\
\hline
\end{tabular}

\subsection{Effects of Pediatric AD on Sleep Duration and Suicidal Ideation of Mothers}

Complex samples logistic regression was conducted to investigate the impact of pediatric AD on sleep duration and suicidal ideation of mothers. Model 1 was a univariate analysis without control variables; model 2 comprised general characteristics (age, marital status, educational level, household income level and occupation) as control variables; and, model 3 included the control variables of model 2 as well as subjects' health-related traits (drinking, smoking, subjective health status, hypertension, hyperlipidemia, asthma, depression, AD, allergic rhinitis and stress perception rate).

Sleep duration was statistically and significantly lower in mothers of children with AD than in mothers of children without AD by $1.477(\mathrm{p}=.044)$ in Model 1, $1.552(\mathrm{p}=.025)$ in Model 2 and $1.547(\mathrm{p}=.025)$ in Model 3. Although not statistically significant, suicidal ideation was higher in mothers of affected children than in mothers of non-affected children by $1.348(\mathrm{p}=.319)$ in Model 1, $1.414(\mathrm{p}=.252)$ in Model 2 and $1.109(\mathrm{p}=.751)$ in Model 3 (Table 4). 
Table 4. The effect of atopic dermatitis in children on sleep duration and suicidal ideation of mothers $(N=2,845)$

\begin{tabular}{llll}
\hline Characteristics & Non-atopic group $(n=2,403)$ & Atopic group $(n=442)$ \\
OR $(p)$ & OR $(p)$ \\
\hline \multirow{3}{*}{ Sleep duration } & Model 1 & 1.0 (reference) & $1.477(.044)$ \\
\cline { 2 - 4 } & Model 2 & 1.0 (reference) & $1.552(.025)$ \\
\cline { 2 - 4 } Suicidal ideation & Model 3 & 1.0 (reference) & $1.547(.025)$ \\
\cline { 2 - 4 } & Model 1 & 1.0 (reference) & $1.348(.319)$ \\
\cline { 2 - 4 } & Model 3 & $1.0($ reference) & $1.414(.252)$ \\
\hline
\end{tabular}

Note. Model 1: No control variables. Model 2: General characteristics (age, marital status, education, household income, occupation) includes control variables. Model 3: Model 2 control variables + Health related characteristics (drinking, smoking, self-reported health status, hypertension, hyperlipidemia, asthma, depression, atopic dermatitis, allergic rhinitis, stress) include control variables.

\section{Discussion}

This study was undertaken to investigate the effects of pediatric $A D$ on sleep duration and suicidal ideation in mothers with children between the ages of 1 and 18 years using the data from the KNHANES VI (2013 and 2015), and was conducted with a large-scale, random sampling method. In the survey, primary caregivers were predominantly mothers. In a previous study (Kim, 2016), the rates of mothers to fathers participating in child caregiving was $79.8 \%$ and $20.2 \%$, indicating that the primary caregivers are usually mothers. For this reason, this study only included mothers as the subjects.

There were no variables showing a significant difference in the general characteristics between $\mathrm{AD}$ and non-AD groups. However, $43.9 \%$ of mothers raised their children alone and $15.6 \%$ were working in the AD group. This information has revealed that further investigation appears to be meaningful to explore parenting trends and social support for mothers responsible for both child-rearing and earning a single income. In regards to health-related traits, the prevalence of asthma, $\mathrm{AD}$, and allergic rhinitis was significantly greater in the $\mathrm{AD}$ group. This outcome implies that genetics is an important risk factor for $\mathrm{AD}$. The rates of developing $\mathrm{AD}$ in children with one atopic parent were $30.7 \%$ in the mother and $22.2 \%$ in the father. These findings demonstrate that children with a family history of both parents with $\mathrm{AD}$ are more prone to $\mathrm{AD}$ and are more likely to be affected by the presence of $\mathrm{AD}$ in the mother rather than the father (Lee, 2003; Kim et al., 2009). Moreover, Pyun (2005) suggested that patients with $\mathrm{AD}$ are more likely to develop respiratory allergic diseases such as asthma or allergic rhinitis. Kim et al. (2009) observed asthma or allergic rhinitis in a large number of mothers with AD. These results are comparable to those of this study. Therefore, knowledge of $\mathrm{AD}$ needs to be deepened in advance by instructing the importance of healthcare management in children with $\mathrm{AD}$ and implementing prenatal and postnatal education programs for mothers with a history of AD.

Common medications used for allergic reactions include steroids, immunosuppressants, antihistamines (Samukawa et al., 2012). Although these types of medications are effective in alleviating symptoms, side effects have been observed in some patients (Boguniewicz \& Leung, 2011). For this reason, further studies are currently in progress to find advanced therapeutic methods for the treatment of allergic diseases.

Of the AD group, mothers with divorced marital status, an educational level of less than elementary school, very low income, occupation, poor subjective health status, hypertension, and a lot of stress were identified to sleep less than 5 hours a day. This outcome implies that mothers were at low socio-economic level and not confident in rating their health status. Parents of children with AD have decreased ability to manage work and family because their night-time sleep is 1-2 hours shorter due to the child's frequent waking from intense itching at night (Moore, 2006; Reid \& Lewis-Jones, 1995). Mothers who experienced sleep disturbances were more likely to have higher levels of anxiety and depression (Moore, 2006). Mothers rearing children with AD are physically exhausted and fatigued due to lack of sleep. They constantly struggle in managing cleaning and cooking for their children during the day and cannot rest or sleep sufficiently in order to prevent their children from scratching at night (Yoo \& Kim, 2006). Chung et al. (2005) reported that mothers of affected children were more likely to experience higher child-rearing 
stress and depression than mothers of non-affected children. Pappard et al. (2000) found out that hypertension rates were higher among subjects with sleep disturbances. Therefore, and by recognizing the need for child-rearing and health status in low-income mothers raising children alone after divorce, primary healthcare centers need to develop and implement intervention programs.

Risk factors of suicidal ideation were marital status (divorced), household income (lower levels), subjective health status (unhealthy), depression, allergic rhinitis, and stress perception rate. According to Kwak et al. (2004), divorce and low socio-economic status were associated with high suicide frequency. Comparable to the results of our study, Choi et al. (2011) determined that suicidal ideation risk was greater in individuals with broken marriages, lower educational levels, lower income levels, poorer subjective health status, depression, and higher stress perception. Furthermore, Lee (2011) revealed that mothers with better health status had higher life satisfaction. Since the physical and mental well-being of mothers is vital for their success in long-term management of chronic conditions, this result implies that social measures are necessary to manage maternal health along with their children affected with AD.

Sleep duration was found to be 1.477 to 1.552 times lower in the AD group when compared to the non-AD group. Parents of children with AD were 4-8 times more likely to sleep less than 6 hours on average, have shorter sleep duration, and be more prone to insomnia when compared to the parents of healthy children, and exhibit clear signs of sleep disturbances (Meltzer \& Booster, 2016). In Lee et al.'s study (2009), consistent short sleep durations of less than 6 hours were associated with increased risk of suicidal ideation. According to Bernert et al. (2005), more severe insomnia symptoms were related to increased levels of suicidal ideation. In our study, no statistical significance was found in suicidal ideation between $\mathrm{AD}$ and non- $\mathrm{AD}$ groups, but suicidal ideation rates were 1.348-1.414 times higher in mothers of affected children than in those of non-affected children. Mothers of AD children aged less than 18 years were more vulnerable to experiencing suicidal impulses by $41 \%$ up to $90 \%$ than those not, indicating higher prevalence of suicidal ideation in the AD group (Choi \& Lee, 2017). Intervention measures appear to be vital for the understanding and easing of emotional problems in mothers with children with $\mathrm{AD}$.

The aims of this study were to investigate the effects of pediatric AD on sleep duration and suicidal ideation of mothers, and provide a baseline from which intervention programs can be developed to promote life satisfaction and emotional well-being in mothers (as primary caregivers) who carry out managing child's conditions and daily child-rearing. These results revealed that mothers with $\mathrm{AD}$ children have shorter sleep duration. Unlike their married counterparts, divorced mothers were more likely to sleep less as they have sole responsibility for raising and caring for their children and managing itching at night. In this study, after controlling for variables of general characteristics, sleep duration was found to be more than 1.5 times lower in the AD group than in the non-AD group. After controlling for all variables of health-related traits, sleep duration was more than 1.5 times lower. Likewise, suicidal ideation was more than 1.4 times higher in the AD group as general characteristics were controlled. We cannot exclude the possibility of secondary depression or suicidal ideation associated with sleep disturbances in mothers with AD children. Results of this study show that timely nursing interventions are warranted to provide physical, mental and social support for mothers and to maintain their health. A systematic method preventing suicide is needed through continuous monitoring of parents with AD children. In addition, we suggest the development of intervention programs for more effective and integrated suicide prevention strategies at the state level.

\section{Competing Interests Statement}

The authors declare that there are no conflicts of interest.

\section{References}

Basra, M. K. A., \& Finlay, A. Y. (2007). The family impact of skin diseases: the Greater Patient concept. British Journal of Dermatology, 156(5), 929-937. https://doi.org/10.1111/j.1365-2133.2007.07794.x

Bernert, R. A., Joiner, T. E., Cukrowicz, K. C., Schmidt, N. B., \& Krakow, B. (2005). Suicidality and sleep disturbances. Sleep, 28(9), 1135-1141. https://doi.org/10.1093/sleep/28.9.1135

Boguniewicz, M., \& Leung, D. Y. (2011). Atopic dermatitis: a disease of altered skin barrier and immune dysregulation. Immunological Reviews, 242(1), 233-246. https://doi.org/10.1111/j.1600-065x.2011. 01027.x

Chamlin, S. L., Frieden, I. J., Williams, M. L., \& Chren, M. M. (2004). Effects of atopic dermatitis on young American children and their families. Pediatrics, 114(3), 607-611. https://doi.org/10.1542/peds.2004-0374.

Choi, H. A., Lee, D. S., \& Kim, Y. J. (2011). Analysis on depression and suicidal ideation of Korean female 
adults-by using Korea national health and nutrition examination survey (KNHANES IV). Journal of The Korean Data Analysis Society, 13(2), 709-720.

Choi, S. M., \& Lee, H. Y. (2017). Impacts of caring for children with atopic dermatitis on mental health of their mothers. Korean Public Health Research, 43(2), 37-52.

Chung, S. W., Chang, E. Y., Lee, H. C., Shin, M. Y., Kim, B. E., Ahn, K. M., \& Lee, S. I. (2005). The relationships among severity score, behavioral problem, parental stress, maternal depression, and social support in children with atopic dermatitis. The Korean Academy of Asthma, Allergy and Clinical Immunology, 25(1), 37-52.

Devereux, G., \& Seaton, A. (2005). Diet as a risk factor for atopy and asthma. Journal of Allergy and Clinical Immunology, 115(6), 1109-1117. https://doi.org/10.1016/j.jaci.2004.12.1139

Draaisma, E., Garcia - Marcos, L., Mallol, J., Solé, D., Pérez - Fernández, V., \& Brand, P. L. (2015). A multinational study to compare prevalence of atopic dermatitis in the first year of life. Pediatric Allergy and Immunology, 26(4), 359-366. https://doi.org/10.1111/pai.12388

Kimata, H. (2006). Prevalence of suicidal ideation in patients with atopic dermatitis. Suicide and Life-Threatening Behavior, 36(1), 120-124. https://doi.org/10.1521/suli.2006.36.1.120

Kim, D. H. (2011). Factors associated with parenting stress of mothers of school-aged children with atopic dermatitis. Stress Research, 19(2), 115-121.

Kim, S. N. (2016). A study on the family support needs due to parenting stress and burden of parents having children with severe brain lesions. Unpublished master's thesis. Kangnam University. Seoul.

Kim, H. Y., Jang, E. Y. Sim, J. H., Kim, J. H., Chung, Y., Park, S. H., \& Lee, S. I. (2009). Effects of family history on the occurrence of atopic dermatitis in infants. Allergy Asthma and Respiratory Disease, 43(2), 106-114.

Korea Statistical Information Service. (2016). 2015 Cause of death statistics. Korea, Seoul: Seoul: Korea Statistical Information Service.

Kwak, K. P., Song, C. J., Sakong, J. K., \& Lee, K. H. (2004). Assessment and prediction of suicide risk. Medical Institute of the Dongguk University, 11(1), 83-94.

Kwon, K. J. (2014, Jan). Killed a daughter who had five years of atopic dermatitis, and killed herself. The Kyunghyang Shinmun.

Lapidus, C. S., \& Kerr, P. E. (2001). Social impact of atopic dermatitis. Medicine and health, 84(9), 294-295.

Lee, S. I. (2003). Common allergic diseases in children. Journal of the Korean Medical Association, 46(3), 231-243. https://doi.org/10.5124/jkma.2003.46.3.231

Lee, Y. S. (2011). The difference of life satisfaction and mental health between mothers of atopic dermatitis child and mothers of normal child. Unpublished master's thesis. Chung-Ang University. Seoul.

Lee, Y. J., Kim, S. J., Cho, I. H., Kim, J. H., Bae, S. M., Koh, S. H., \& Cho, S. J. (2009). A study on the relationship between sleep duration and suicidal idea in an urban area of South Korea. Sleep Medicine and Psychophysiology, 16(2), 85-90.

Lewandowski, A. S., Ward, T. M., \& Palermo, T. M. (2011). Sleep problems in children and adolescents with common medical conditions. Pediatric Clinics of North America, 58(3), 699-713. https://doi.org/10.1016/ j.pcl.2011.03.012

Lifschitz, C. (2015). The impact of atopic dermatitis on quality of life. Annals of Nutrition and Metabolism, 66(1), 34-40. https://doi.org/10.1159/000370226

Meltzer, L. J., \& Booster, G. D. (2016). Sleep disturbance in caregivers of children with respiratory and atopic disease. Journal of Pediatric Psychology, 41(6), 643-650. https://doi.org/10.1093/jpepsy/jsw016

Ministry of Health \& Welfare. (2016). 2015 National Health Statistics I. Korea, Seoul: Ministry of Health \& Welfare.

Moore, K., David, T. J., Murray, C. S., Child, F., \& Arkwright, P. D., (2006). Effect of childhood eczema and asthma on parental sleep and well-being: a prospective comparative study. British Journal of Dermatology, 154(3), 514-518. https://doi.org/10.1111/j.1365-2133.2005.07082.x

National Health Insurance Service. (2016). Major "allergy" disease, child. Adolescence (under 12 years). Korea, Seoul: National Health Insurance Service.

Reid, P., \& Lewis-Jones, M. S. (1995). Sleep difficulties and their management in preschoolers with atopic eczema. 
Clinical and Experimental Dermatology, 20(1), 38-41. https://doi.org/10.1111/j.1365-2230.1995. tb01280.x

Samukawa, K., Izumi, Y., Shiota, M., Nakao, T., Osada-Oka, M., Miura, K., \& Iwao, H. (2012). Red ginseng inhibits scratching behavior associated with atopic dermatitis in experimental animal models. Journal of Pharmacological Sciences, 118(3), 391-400. https://doi.org/10.1254/jphs.11182fp

Peppard, P. E. Young, T., Palta, M., \& Skatrud, J. (2000). Prospective study of the association between sleep-disordered breathing and hypertension. New England Journal of Medicine, 342(19), 1378-1384. https://doi.org/10.1056/NEJM200005113421901

Pyun, B. Y. (2005). Risk factors of atopic dermatitis in young children. Pediatric Allergy and Respiratory Disease, 15(3), 235-237.

van Oers, H. A., Haverman, L., Limperg, P. F., van Dijk-Lokkart, E. M., Maurice-Stam, H., \& Grootenhuis, M. A. (2014). Anxiety and depression in mothers and fathers of a chronically ill child. Maternal and Child Health Journal, 18(8), 1993-2002. https://doi.org/10.1007/s10995-014-1445-8

Warschburger, P., Buchholz, H., \& Petermann, F. (2004). Psychological adjustment in parents of young children with atopic dermatitis: which factors predict parental quality of life?. British Journal of Dermatology, 150(2), 304-311. https://doi.org/10.1111/j.1365-2133.2004.05743.x

Williams, H., Robertson, C., Stewart, A., Aït-Khaled, N., Anabwani, G., Anderson, I. R., \& Weiland, S. K. (1999). Worldwide variations in the prevalence of symptoms of atopic eczema in the international study of asthma and allergies in childhood. Journal of Allergy and Clinical Immunology, 103(1), 125-138. https://doi.org/10.1016/S0091-6749(99)70536-1

Yoo, I. Y., \& Kim, D. H. (2004). Parenting stress and needs of mothers of children with atopic dermatitis. Child Health Nursing Research, 10(4), 423-430.

Yoo, I. Y., \& Kim, D. H. (2006). Mothers'experiences of parenting of a child with atopic dermatitis. Journal of the Korean Society of Maternal and Child Health, 10(2), 169-179.

Yoon, J. H., \& Bae, J. H. (2013). Difficulties and needs of parents raising young children with atopic dermatitis. The Journal of Eco-Early Childhood Education, 12(1), 113-137.

\section{Copyrights}

Copyright for this article is retained by the author(s), with first publication rights granted to the journal.

This is an open-access article distributed under the terms and conditions of the Creative Commons Attribution license (http://creativecommons.org/licenses/by/4.0/). 